\title{
Transcriptomic changes induced by de-activation of lower glycolysis and its advantage on pentose sugar metabolism in Saccharomyces cerevisiae
}

\author{
Minhye Shin ${ }^{1}$, Heeyoung Park ${ }^{2}$, Sooah $\mathrm{Kim}^{3}$, Eun Joong Oh $\mathrm{Oh}^{4}$, Deokyeol Jeong ${ }^{2}$, \\ Clarissa Florencia ${ }^{5}$, Kyoung Heon Kim ${ }^{1}$, Yong-Su Jin ${ }^{5}$, and Soo Rin Kim² \\ ${ }^{1}$ Korea University \\ ${ }^{2}$ Kyungpook National University \\ ${ }^{3}$ Jeonju University \\ ${ }^{4}$ University of Colorado Boulder \\ ${ }^{5}$ University of Illinois at Urbana-Champaign
}

March 22, 2021

\begin{abstract}
As a microbial host for cellulosic biofuel production, Saccharomyces cerevisiae needs to be engineered to express a heterologous xylose pathway. However, it has been challenging to optimize the engineered strain for efficient and rapid fermentation of xylose. Deletion of PHO13 (pho13) has been reported to be a crucial genetic perturbation for improving xylose fermentation. A confirmed mechanism of the pho13-positive effect on xylose fermentation is that the deletion of PHO13 transcriptionally activates the genes in the non-oxidative pentose phosphate pathway (PPP). In the present study, we reported that a pho13positive effect was not observed from a couple of engineered strains, among the many others we have examined. To extend our knowledge of pho13-mediated metabolic regulation, we performed genome sequencing of pho13-negative strains. We identified a loss-of-function mutation in GCR2 responsible for the pho13-negative phenotype. Gcr2 is a transcriptional activator of the lower glycolytic pathway. Thus, the deletion of GCR2 (gcr2) led to deactivation of lower glycolysis as confirmed by RNA-seq. Also, gcr2 resulted in the up-regulation of PPP genes, which explains the improved xylose fermentation of gcr2 mutants. As pho13 and gcr2 cause similar transcriptional changes with PPP genes, there was no synergistic effect between pho13 and gcr2 for improving xylose fermentation. The present study identified GCR2 as a new knockout target to improve xylose fermentation and cellulosic biofuel production.
\end{abstract}

Now published in Frontiers in Bioengineering and Biotechnology doi: 10.3389/fbioe.2021.654177

\section{Introduction}

Cellulosic biofuels are renewable liquid-fuel alternatives due to abundant feedstock availability and substantial $\mathrm{CO}_{2}$ emission reduction (Lynd, 2017). Saccharomyces cerevisiae plays an essential role in production of cellulosic biofuels by fermenting cellulosic sugars, mainly glucose and xylose, which requires engineering of the yeast via a heterologous xylose pathway (Kim et al., 2013c; Richa et al., 2019). Current efforts on the metabolic engineering of the yeast are still focused on improving the xylose fermentation yield and productivity under multiple stress conditions of lignocellulosic biomass hydrolysates (Park et al., 2020; Qin et al., 2020).

Previously, an efficient xylose-fermenting strain of S. cerevisiae (SR8) was developed through the introduction of a heterologous xylose pathway, optimization of its expression levels, and adaptive evolution, which resulted in a loss-of-function mutation on PHO13 (Kim et al., 2013d). Continued efforts have discovered that the deletion of PHO13 (pho13) resulted in transcriptional and metabolic changes favorable to xylose and other C5 sugar fermentation (Kim et al., 2015; Xu et al., 2016; Ye et al., 2019). However, as PHO13 was first 
discovered as a knockout target to improve xylose fermentation (Ni et al., 2007; Van Vleet et al., 2008), detailed molecular mechanisms underlying the pho13-phositive phenotype remained unelucidated. The most advanced finding thus far is that pho13 results in the transcriptional activation of non-oxidative pentose phosphate pathway (PPP) genes, which therefore facilitates xylose metabolism (Xu et al., 2016).

However, through this study pho13-positive effect on xylose fermentation was seen as strain backgrounddependent, and one factor is associated with a loss of function mutation in GCR2 coding for a transcriptional activator of the genes in glycolysis. Gcr2 enhances the CT box-dependent transcriptional activation of a Rap1-Gcr1 complex required for the expression of glycolytic genes (Huie et al., 1992). As a transcriptional activation of complex, Rap1 and Gcr1 provide the specific DNA-binding and the activation of glycolytic and ribosomal genes, respectively (Sasaki et al., 2005; Uemura and Fraenkel, 1990). Function of Gcr2 is to provide an activation domain to the Gcr1p-Gcr2p complex mediating high level of glycolytic gene expression (Uemura and Jigami, 1992). However, it is unknown how the regulatory systems would function if new foreign pathways, such as the heterologous xylose-assimilating genes, are introduced.

Therefore, the aim of the current study was to understand the strain specific pho13-positive effect by investigating transcriptomic changes of gcr2 mutant during glucose and xylose fermentation. The result suggests that the native regulator system, primarily the transcriptional regulations, is highly associated with the suboptimal xylose fermentation by xylose-fermenting $S$. cerevisiae.

\subsection{Strain construction}

All $S$. cerevisiae strains used in the present study are listed in Table 1 . To construct xylose-fermenting strains, the linear expression cassette of Scheffersomyces stipitis XYL1, XYL2 , and XYL3 genes was used as described previously (Kim et al., 2013d). To constructpho13 mutants of the xylose-fermenting strains, thepho13 ::KanMX4 cassette was used as described previously (Kim et al., 2013d). To isolate spores from the KSM diploid strain, tetrad dissection was performed as described previously (Kim et al., 2017). To construct the gcr2 mutant, the gcr2 ::KanMX4 cassette was amplified from the genomic DNA of the BY4742 gcr2 strain (clone ID: 12013) of the Yeast Knockout Collection (Thermo Fisher Scientific, USA) by polymerase chain reaction (PCR) using SOO303/298 primers. The PCR product was purified and genome-integrated to the SR7 strain by the LiAc transformation method (Gietz and Schiestl, 2007). The resulting deletion mutant was selected on an agar medium containing $10 \mathrm{~g} / \mathrm{L}$ yeast extract, $20 \mathrm{~g} / \mathrm{L}$ peptone, $20 \mathrm{~g} / \mathrm{L}$ glucose (YPD), $15 \mathrm{~g} / \mathrm{L}$ agar, and $300 \mathrm{mg} / \mathrm{mL}$ G418 sulfate (GoldBio, St. Louis, MO, USA).

\subsection{Culture conditions}

A colony of yeast cells was pre-cultured in $5 \mathrm{~mL}$ YPD for $24 \mathrm{~h}$ at $30^{\circ} \mathrm{C}$ and $250 \mathrm{rpm}$. The culture containing 25 or $2.5 \mathrm{mg}$ cells was centrifuged at $15,000 \mathrm{rpm}$ for $1 \mathrm{~min}$ at $4^{\circ} \mathrm{C}$. The cells were resuspended in $50 \mathrm{~mL} \mathrm{YPD}$ (40 g/L glucose) or YPX (40 g/L xylose) in a $250 \mathrm{~mL}$ Erlenmeyer flask, and the culture with an initial cell density of 0.5 or $0.05 \mathrm{~g} / \mathrm{L}$ was incubated at $30^{\circ} \mathrm{C}$ and $80 \mathrm{rpm}$. All experiments were done in triplicated.

\subsection{Fermentation profiles}

Cell growth was monitored at $600 \mathrm{~nm}$ using a spectrophotometer $\left(\mathrm{OD}_{600}\right)$. The concentrations of substrates and metabolites were determined by high-performance liquid chromatography (Agilent Technologies 1260 Series, Santa Clara, CA, USA) equipped with a refractive index detector using a Rezex ROA-Organic Acid $\mathrm{H}^{+}(8 \%)$ column (Phenomenex, Inc., Torrance, CA, USA). The column was eluted with $0.005 \mathrm{~N} \mathrm{H}_{2} \mathrm{SO}_{4}$ at a flow rate of $0.6 \mathrm{~mL} / \mathrm{min}$ and $50^{\circ} \mathrm{C}$.

\subsection{Genome sequencing}

For single nucleotide polymorphism (SNP) discovery in thepho13 -negative strain (YSX3), the genome of the YSX3 strain and its parental strain (L2612) were re-sequenced as described previously (Kim et al., 2013d). Briefly, genomic DNA was prepared by YeaStar Genomic DNA Kit (Zymo Research), and the DNA quality was evaluated on a $1 \%$ agarose gel. The genomic DNA samples were then sequenced using an Illumina HiSeq 2000 system, and the sequencing results were analyzed using the CLC Genomic Workbench (version 6.5) 
program. Among the 46 SNPs identified, 44 SNPs were found to be non-synonymous, and only 2 SNPs in YEN1 and GCR2 genes were confirmed in the YSX3 strain by Sanger sequencing. The two SNPs were also confirmed.

\subsection{RNA-seq}

For transcriptomic analysis, RNA-seq was performed as described previously (Kim et al., 2015). Briefly, RNA was extracted from exponentially growing $0.5 \mathrm{mg}$ cells of the control strain (SR7) and gcr2 mutant (SR7 gcr2 ) on glucose or xylose using a Qiagen RNeasy Mini Kit, and the RNA quality was evaluated using a Bioanalyzer RNA chip. The samples with high-quality total RNA were sequenced using an Illumina HiSeq 2000 system. The sequencing results were then analyzed using the CLC Genomic Workbench (version 6.5) to investigate RNA-seq quality, differentially expressed (DE) genes, and Gene Set Enrichment Analysis (GSEA). Fold changes were calculated based off the total number of exon reads per kilobase of exon length per million mapped reads (RPKM) between SR7 and SR7 gcr2 strains.

\section{Results}

\section{1. pho13-negative phenotype was found in a few xylose-fermenting engineered strains}

As reported previously, pho13 improves the xylose fermentation capability of engineered strains of $S$. cerevisiae(Ni et al., 2007; Van Vleet et al., 2008), and other studies have confirmed it with different strain backgrounds (Fujitomi et al., 2012; Jeong et al., 2020; Kim et al., 2013d). Although the detailed molecular mechanism is still unknown, pho13 results in the transcriptional activation of the genes in non-oxidative PPP (Kim et al., 2015; Ye et al., 2019) and reduction of the dephosphorylation product of sedoheptulose-7phosphate, possibly suggesting the phosphatase activity of Pho13 (Xu et al., 2016). To further explorepho13 -mediated metabolic regulation, the pho13 effect was tested with a broader range of strains that we have constructed, as listed in Fig. 1a and Table 1. When a plasmid expressing heterologous xylose pathway (XYL1 , XYL2 , and XYL3 derived fromS. stipitis ) was introduced to four different strain backgrounds, all of the resulting strains (DX123, JX123, CX123, and LX123) and their derivatives (SR6, SR7, and DGX23) showed an improved xylose consumption rate by pho13. However, two strains, YSX3 and its derivative (DA24), which were constructed independently from LX123 strain, did not show any improvement by pho13. Because the YSX3 strain underwent multiple transformations and vigorous screening processes for strain optimization previously (Jin et al., 2003), we suspected that some unknown mutations were acquired by chance.

\subsection{Loss-of-function mutation in GCR2 is responsible for the pho13-negative phenotype}

To identify the molecular mechanism of the pho13 -negative phenotype of the YSX3 strain, genome sequencing of the YSX3 strain and its parental strain (L2612) was performed, and non-synonymous SNPs in YEN1 and GCR2 were identified (Fig. 1b). From the diploid of YSX3 and a D452-2 derivative, four haploid spores were dissected out, and two spores showed improved xylose fermentation by pho13, whereas the other two spores did not. Sanger sequencing of the spores revealed that both pho13 -positive spores had wild-type GCR2 , whereas pho13 -negative spores had the mutant GCR2 gene. Because the mutation in GCR2 resulted in the truncation of the protein (Glu204*), we assumed its loss-of-function mutation. When the deletion of GCR2 (gcr2 ) was tested in the SR7 strain, gcr2 mutant showed faster xylose consumption and higher ethanol yield compared to the control strain, but the double deletion of gcr2 and pho13 was not synergistic (Fig. 1c-e). These results suggested that the loss-of-function mutation in GCR2 is responsible for the pho13 -negative phenotype of the YSX3 strain. Also, it can be concluded that GCR2 is a novel deletion target to improve xylose fermentation.

\subsection{Global transcriptional changes induced by gcr2}

GCR2 encodes a transcriptional activator of glycolytic genes; therefore, its deletion leads to the transcriptional down-regulation of glycolytic genes and up-regulation of citric acid cycle genes during glucose metabolism (Fendt et al., 2010; Sasaki and Uemura, 2005; Uemura and Jigami, 1992). To investigate the transcriptional changes by gcr2 during xylose metabolism, S. cerevisiae SR7 and SR7 gcr2 strains grown on glucose or xylose 
were subjected to RNA-seq, and high-quality sequencing data were obtained (Table 2). Hierarchical clustering and multivariate analysis based on Pearson's correlation and principal component analysis, respectively, indicated that the transcriptomic profiles of glucose and xylose metabolism were the primary determinants (Fig. 2). Notably,gcr2 mutant samples were clustered separately from control samples on both glucose and xylose fermentation conditions, suggesting global transcriptional changes evoked by gcr2 regardless of the type of substrate. Meanwhile, the number of DE genes in the gcr2mutant compared to the control strain $(p$ $<0.05,>2$-fold) was 1638 and 605 on glucose and xylose, respectively. Also, the most significant DE genes in the gcr2mutant compared to the control strain $(p<0.01,>10$-fold) were 17 and 5 on glucose and xylose, respectively (Table 3 ). The larger number of DE genes and the more significant fold changes under glucose conditions suggest that Gcr2 is responsible for more global transcriptional regulation of glucose metabolism compared to xylose metabolism.

\subsection{GSEA of DE genes by gcr2}

DE genes in the gcr2 mutant were subjected to GSEA using Gene Ontology (GO) biological process (Table 4). On both glucose and xylose conditions, genes associated with translation, nucleotide biosynthesis, lipid biosynthesis, and one-carbon metabolism were up-regulated, and genes associated with protein transport were down-regulated. However, the direction of the transcriptional changes by gcr2 in two gene sets (sugar metabolism and oxidation-reduction) and their genes were opposites depending on the type of substrates; they were up-regulated on xylose but down-regulated on glucose (Fig. 3). For example, ALD3, encoding aldehyde dehydrogenase, is known to be induced in response to stress; it was induced by gcr2 under xylose conditions but repressed under glucose conditions. Because Gcr2 is a native transcriptional activator for glucose metabolism, the heterologous xylose metabolism might interfere with the native metabolic regulation and cause some discrepancies in the direction of transcriptional regulation mediated by Gcr2.

\subsection{Transcriptional changes in central metabolic pathways induced by gcr2}

To better understand the effect of gcr2 on xylose metabolism, the fold changes of DE genes in the glycolytic pathway, the PPP, and the citric acid cycle were systematically compared between glucose and xylose conditions (Fig. 4). Two significant transcriptional changes were observed on both glucose and xylose conditions. First, gcr2 led to the down-regulation of some glycolytic genes, most critically the GPM1 gene, encoding phosphoglycerate mutase, which is a key enzyme of the lower glycolic pathway. Second, gcr2 up-regulated non-oxidative PPP genes, most critically the TAL1 gene, encoding transaldolase. However, in greater detail, gcr2 -mediated transcriptional changes in central metabolic pathways were more prominent during xylose metabolism. In addition to GPM1 gene,TDH2, ENO1, and CDC19 genes in the lower glycolytic pathway were significantly down-regulated only under xylose conditions. Moreover, SOL4, GND2, and TKL2 genes in oxidative and non-oxidative PPP were up-regulated considerably only under xylose conditions.

\section{Discussion}

In the present study, GCR2 encoding a transcriptional activator of glycolytic genes was identified as a novel deletion target ( gcr2 ) to improve the xylose fermentation of $S$. cerevisiaeexpressing a heterologous xylose pathway. RNA-seq results revealed thatgcr2 results in not only the down-regulation of glycolytic genes but also the up-regulation of PPP genes, which explains the improved xylose metabolism by gcr2 . Specifically, gcr2 triggers the up-regulation of oxidative and non-oxidative PPP genes contributing to NADPH production for $\mathrm{NAD}(\mathrm{P}) \mathrm{H}$-specific xylose reductase $(X Y L 1)$ and direct metabolism of xylose.

Deleting both pho13 and gcr2 did not synergistically accelerate xylose fermentation. This outcome leads to a hypothesis that both genes might share similar molecular mechanism. Indeed, considering previous studies on pho13 (Kim et al., 2015; Xu et al., 2016), bothpho13 and gcr2 result in the up-regulation of TAL1 gene, the essential overexpression target to improve xylose fermentation, as well as other genes in PPP. However, gcr2results in more global transcriptional changes compared to pho13. The number of DE genes by pho13 was 12 and 277 on glucose and xylose, respectively, which was one order of magnitude lower than that by gcr2. Also, some transcriptional changes induced bygcr2 were opposite directions from that by pho13 ; especially, genes in the lower glycolytic pathway were repressed by gcr2 but activated by pho13 during xylose 
fermentation. Therefore, it is difficult to ignore the possibility that pho13 - and gcr2 -mediated metabolic regulation are independent of each other but share TAL1 activation by chance.

Some native regulatory systems of $S$. cerevisiae might act negatively to heterologous metabolism. However, it is challenging to systematically investigate all native regulatory genes to identify inhibitory ones toward introduced pathways. One of the most practical solutions for metabolic engineering is to use adaptive evolution to induce spontaneous mutations favorable to heterologous metabolism. Identification of $\mathrm{PHO} 13$ and GCR2 is a successful example of such metabolic engineering strategy. Assisted with genome sequencing and omics approaches, such as RNA-seq, spontaneous mutations inPHO13 (intended) or GCR2 (by chance) were identified independently and led to the discovery of native inhibitory factors against the heterologous xylose pathway in our previous and present studies.

\section{Acknowledgments}

This work was supported by a National Research Foundation of Korea (NRF) grant (2019R1F1A1062633) funded by the Korea Government (MSIT).

\section{Author contributions}

YSJ and SRK designed the experiments. MS, HP, EJO, DJ, and CF carried out the experiments. SK and KHK performed the statistical analysis of RNA-seq data. MS, YSJ, and SRK drafted and finalized the manuscript. All authors contributed to the final manuscript. All authors read and approved the final manuscript.

\section{Data availability}

The datasets supporting the conclusion of this article are included in the article.

\section{Conflict of interest}

The authors declare that they have no conflict of interest.

\section{Ethical approval}

This article does not contain any studies with human participants or animals performed by any of the authors.

\section{References}

Fendt, S.-M., Buescher, J. M., Rudroff, F., Picotti, P., Zamboni, N., Sauer, U., 2010. Tradeoff between enzyme and metabolite efficiency maintains metabolic homeostasis upon perturbations in enzyme capacity. Molecular Systems Biology. 6,356.

Fujitomi, K., Sanda, T., Hasunuma, T., Kondo, A., 2012. Deletion of the PHO13 gene inSaccharomyces cerevisiae improves ethanol production from lignocellulosic hydrolysate in the presence of acetic and formic acids, and furfural. Bioresource Technology. 111, 161-166.

Gietz, R. D., Schiestl, R. H., 2007. High-efficiency yeast transformation using the LiAc/SS carrier DNA/PEG method. Nature Protocols. 2, 31-34.

Ha, S.-J., Galazka, J. M., Rin Kim, S., Choi, J.-H., Yang, X., Seo, J.-H., Louise Glass, N., Cate, J. H. D., Jin, Y.-S., 2011. Engineered Saccharomyces cerevisiae capable of simultaneous cellobiose and xylose fermentation. Proceedings of the National Academy of Sciences. 108, 504-509.

Ha, S.-J., Kim, S. R., Kim, H., Du, J., Cate, J. H. D., Jin, Y.-S., 2013. Continuous co-fermentation of cellobiose and xylose by engineered Saccharomyces cerevisiae . Bioresource Technology. 149, 525-531.

Huie, M. A., Scott, E. W., Drazinic, C. M., Lopez, M. C., Hornstra, I. K., Yang, T. P., Baker, H. V., 1992. Characterization of the DNA-binding activity of GCR1: in vivo evidence for two GCR1-binding sites in the upstream activating sequence of TPI ofSaccharomyces cerevisiae. Molecular and Cellular Biology. 12, 2690-2700. 
Jeong, D., Oh, E. J., Ko, J. K., Nam, J.-O., Park, H.-S., Jin, Y.-S., Lee, E. J., Kim, S. R., 2020. Metabolic engineering considerations for the heterologous expression of xylose-catabolic pathways in Saccharomyces cerevisiae. PLOS ONE. 15, e0236294.

Jin, Y.-S., Ni, H., Laplaza, J. M., Jeffries, T. W., 2003. Optimal growth and ethanol production from xylose by recombinant Saccharomyces cerevisiae require moderate d-xylulokinase activity. Applied and Environmental Microbiology. 69, 495-503.

Kim, S. R., Ha, S.-J., Kong, I. I., Jin, Y.-S., 2012. High expression of XYL2 coding for xylitol dehydrogenase is necessary for efficient xylose fermentation by engineered Saccharomyces cerevisiae. Metabolic Engineering. 14, 336-343.

Kim, S. R., Kwee, N. R., Kim, H., Jin, Y.-S., 2013a. Feasibility of xylose fermentation by engineeredSaccharomyces cerevisiae overexpressing endogenous aldose reductase (GRE3), xylitol dehydrogenase (XYL2), and xylulokinase (XYL3) from Scheffersomyces stipitis . FEMS Yeast Research. 13, 312-321.

Kim, S. R., Lee, K.-S., Kong, I. I., Lesmana, A., Lee, W.-H., Seo, J.-H., Kweon, D.-H., Jin, Y.-S., 2013b. Construction of an efficient xylose-fermenting diploidSaccharomyces cerevisiae strain through mating of two engineered haploid strains capable of xylose assimilation. Journal of Biotechnology. 164, 105-111.

Kim, S. R., Park, Y.-C., Jin, Y.-S., Seo, J.-H., 2013c. Strain engineering of Saccharomyces cerevisiaefor enhanced xylose metabolism. Biotechnology Advances. 31,851-861.

Kim, S. R., Skerker, J. M., Kang, W., Lesmana, A., Wei, N., Arkin, A. P., Jin, Y.-S., 2013d. Rational and evolutionary engineering approaches uncover a small set of genetic changes efficient for rapid xylose fermentation in Saccharomyces cerevisiae. PLOS ONE. 8, e57048.

Kim, S. R., Skerker, J. M., Kong, I. I., Kim, H., Maurer, M. J., Zhang, G.-C., Peng, D., Wei, N., Arkin, A. P., Jin, Y.-S., 2017. Metabolic engineering of a haploid strain derived from a triploid industrial yeast for producing cellulosic ethanol. Metabolic Engineering. 40, 176-185.

Kim, S. R., Xu, H., Lesmana, A., Kuzmanovic, U., Au, M., Florencia, C., Oh, E. J., Zhang, G., Kim, K. H., Jin, Y.-S., 2015. Deletion of PHO13, encoding haloacid dehalogenase type IIA phosphatase, results in upregulation of the pentose phosphate pathway in Saccharomyces cerevisiae. Applied and Environmental Microbiology. 81, 1601-1609.

Lynd, L. R., 2017. The grand challenge of cellulosic biofuels. Nature Biotechnology. 35,912-915.

Ni, H., Laplaza, J. M., Jeffries, T. W., 2007. Transposon mutagenesis to improve the growth of recombinantSaccharomyces cerevisiae on d-Xylose. Applied and Environmental Microbiology. 73, 2061-2066.

Park, H., Jeong, D., Shin, M., Kwak, S., Oh, E. J., Ko, J. K., Kim, S. R., 2020. Xylose utilization inSaccharomyces cerevisiae during conversion of hydrothermally pretreated lignocellulosic biomass to ethanol. Applied Microbiology and Biotechnology. 104, 3245-3252.

Qin, L., Dong, S., Yu, J., Ning, X., Xu, K., Zhang, S.-J., Xu, L., Li, B.-Z., Li, J., Yuan, Y.-J., Li, C., 2020. Stress-driven dynamic regulation of multiple tolerance genes improves robustness and productive capacity of Saccharomyces cerevisiae in industrial lignocellulose fermentation. Metabolic Engineering. 61, 160-170.

Richa, A., lt, sup, gt, Shuvashish, B., Nilesh Kumar, S., and Sachin, K., 2019. Evaluating the pathway for co-fermentation of glucose and xylose for enhanced bioethanol production using flux balance analysis. Biotechnol. Bioprocess Eng. 24,924-933.

Sasaki, H., Kishimoto, T., Mizuno, T., Shinzato, T., Uemura, H., 2005. Expression of GCR1 , the transcriptional activator of glycolytic enzyme genes in the yeastSaccharomyces cerevisiae, is positively autoregulated by Gcr1p. Yeast. 22, 305-319.

Sasaki, H., Uemura, H., 2005. Influence of low glycolytic activities in gcr1 and gcr2mutants on the expression of other metabolic pathway genes inSaccharomyces cerevisiae. Yeast. 22, 111-127. 
Uemura, H., Fraenkel, D. G., 1990.gcr2 , a new mutation affecting glycolytic gene expression inSaccharomyces cerevisiae. Molecular and Cellular Biology. 10, 6389-6396.

Uemura, H., Jigami, Y., 1992. Role of GCR2 in transcriptional activation of yeast glycolytic genes. Molecular and Cellular Biology. 12, 3834-3842.

Van Vleet, J. H., Jeffries, T. W., Olsson, L., 2008. Deleting the para-nitrophenyl phosphatase (pNPPase),PHO13, in recombinant Saccharomyces cerevisiae improves growth and ethanol production on d-xylose. Metabolic Engineering. 10, 360-369.

Xu, H., Kim, S., Sorek, H., Lee, Y., Jeong, D., Kim, J., Oh, E. J., Yun, E. J., Wemmer, D. E., Kim, K. H., Kim, S. R., Jin, Y.-S., 2016. PHO13 deletion-induced transcriptional activation prevents sedoheptulose accumulation during xylose metabolism in engineered Saccharomyces cerevisiae. Metabolic Engineering. 34, 88-96.

Ye, S., Jeong, D., Shon, J. C., Liu, K.-H., Kim, K. H., Shin, M., Kim, S. R., 2019. Deletion of PHO13improves aerobic l-arabinose fermentation in engineeredSaccharomyces cerevisiae. Journal of Industrial Microbiology \& Biotechnology. 46, 1725-1731.

Table 1. Strains and primers used in this study

\begin{tabular}{|c|c|c|}
\hline $\begin{array}{l}\text { Strains or plasmids } \\
\text { Strains }\end{array}$ & Relevant genotype or descriptions & References \\
\hline DX123 & D452-2 XYL1 XYL2 XYL3 & (Kim et al., 2012) \\
\hline DX123 pho13 & DX123 pho13 $\Delta::$ KanMX4 & (Kim et al., 2013d) \\
\hline SR6 & DX123 XYL1 & (Kim et al., 2013d) \\
\hline SR6 pho13 & SR6 pho13 $\Delta::$ KanMX4 & (Kim et al., 2013d) \\
\hline SR7 & SR6 $X Y L 2 X Y L 3$ & (Kim et al., 2013d) \\
\hline SR7 pho13 & SR7 pho13 $\Delta::$ KanMX4 & (Kim et al., 2013d) \\
\hline DGX23 & D452-2 GRE3 XYL2 XYL3 & (Kim et al., 2013a) \\
\hline DGX23 pho13 & DGX23 pho13 $\Delta:: K a n M X 4$ & This study \\
\hline JX123 & JAY291 XYL1 XYL2 XYL3 & (Ha et al., 2013) \\
\hline JX123 pho13 & pho13 $\Delta:: K a n M X 4$ & This study \\
\hline CX123 & CEN.PK 2-1D XYL1 XYL2 XYL3 & This study \\
\hline CX123 pho13 & CX123 pho13 $\Delta::$ KanMX4 & This study \\
\hline LX123 & L2612 XYL1 XYL2 XYL3 & This study \\
\hline LX123 pho13 & LX123 pho13 $\Delta::$ KanMX4 & This study \\
\hline YSX3 & L2612 XYL1 XYL2 XYL3 & (Jin et al., 2003) \\
\hline YSX3 pho13 & pho13 $\Delta::$ KanMX4 & This study \\
\hline DA24 & YSX3 mXYL1 & (Ha et al., 2011) \\
\hline DA24 pho13 & DA24 pho13s::KanMX4 & This study \\
\hline SX3-2 & D452-2 MATa mXYL1 XYL2 XYL3 & (Kim et al., 2013b) \\
\hline $\mathrm{KSM}$ & A diploid strain of YSX3 and SX3-2 & (Kim et al., 2013b) \\
\hline BY4742 gсr2 & Yeast Knockout Collection & Thermo Fisher Scientific \\
\hline SR7 gcr2 & SR7 $\gamma s \rho 2 \Delta::$ KanMX4 & This study \\
\hline \multicolumn{3}{|l|}{ Primers } \\
\hline SOO303 & CAACCCTATGCTACAAGAGCAG & GCR2 upstream \\
\hline SOO298 & CGACACTAAACCCAGCTAACTC & GCR2 downstream \\
\hline
\end{tabular}

Table 2. Summary of RNA-seq quality, read counts, mapping rates and transcript assemblies

\begin{tabular}{lllll}
\hline Strains and conditions & Sample name & Read count & Mapped \% & Mapped to genes \% \\
SR7 in glucose & G1 & $14,962,297$ & 98.6 & 83.6
\end{tabular}




\begin{tabular}{lllll} 
& G2 & $13,540,372$ & 98.6 & 83.4 \\
SR7 gcr2 in glucose & G3 & $13,436,944$ & 97.7 & 84.1 \\
& G1_gcr2 & $13,914,980$ & 98.1 & 83.9 \\
& G2_gcr2 & $13,788,675$ & 97.9 & 83.4 \\
SR7 in xylose & G3_gcr2 & $13,866,059$ & 98.0 & 84.2 \\
& X1 & $15,347,444$ & 96.9 & 81.8 \\
SR7 gcr2 in xylose & X2 & $14,119,100$ & 96.6 & 81.3 \\
& X3 & $13,889,475$ & 96.3 & 80.8 \\
& X1_gcr2 & $13,711,359$ & 96.6 & 83.2 \\
& X2_gcr2 & $13,158,020$ & 96.7 & 84.4 \\
\hline
\end{tabular}

Table 3. Most significant DE genes bygcr2a

\begin{tabular}{|c|c|c|c|c|c|c|}
\hline & Gene name & Fold change & $p$ & $\begin{array}{l}\text { RPKM }^{\mathrm{b}} \\
\text { SR7 }\end{array}$ & $\begin{array}{l}\mathrm{RPKM}^{\mathrm{b}} \\
\text { SR7 gcr2 }\end{array}$ & Molecular functions \\
\hline \multirow[t]{17}{*}{ Glucose } & $I M D 2$ & 62.2 & $3.1 \mathrm{E}-05$ & $19.6 \pm 3.5$ & $1217.9 \pm 99.4$ & IMP dehydrogenase activity \\
\hline & $D B P 2$ & 16.9 & $1.6 \mathrm{E}-04$ & $20.9 \pm 15.1$ & $353.7 \pm 39.3$ & ATP binding \\
\hline & NOPr & 14.5 & 8.0E-05 & $17.6 \pm 8.7$ & $254.9 \pm 23.4$ & $\mathrm{~ns}$ \\
\hline & $T I P 1$ & 12.7 & $9.6 \mathrm{E}-06$ & $158.4 \pm 5.4$ & $2009.7 \pm 114.1$ & Structural constituent of cell wall \\
\hline & $R P S 26 B$ & 11.1 & $1.3 \mathrm{E}-05$ & $113.0 \pm 30.8$ & $1258.2 \pm 69.4$ & Structural constituent of ribosome \\
\hline & $C G R 1$ & 11.1 & $3.3 \mathrm{E}-05$ & $17.1 \pm 11.6$ & $189.6 \pm 8.9$ & ns \\
\hline & $N S R 1$ & 10.5 & $2.1 \mathrm{E}-04$ & $33.3 \pm 20.6$ & $350.1 \pm 37.5$ & DNA binding \\
\hline & GUA1 & 10.2 & $4.2 \mathrm{E}-05$ & $47.1 \pm 23.6$ & $482.5 \pm 31.0$ & GMP synthase \\
\hline & $H X T 5$ & -10.2 & $3.9 \mathrm{E}-03$ & $562.3 \pm 146.1$ & $55.1 \pm 12.1$ & Glucose transmembrane transporter \\
\hline & $Y M L 131 W$ & -10.4 & 7.3E-04 & $1401.5 \pm 231.0$ & $135.3 \pm 39.1$ & Oxidoreductase activity \\
\hline & $t L(C A A) G 1$ & -10.7 & $5.4 \mathrm{E}-03$ & $15.0 \pm 4.3$ & $1.4 \pm 0.6$ & Triplet codon-amino acid adaptor ac \\
\hline & GND2 & -11.3 & $1.1 \mathrm{E}-03$ & $142.1 \pm 26.5$ & $12.6 \pm 2.1$ & Phosphogluconate dehydrogenase \\
\hline & $Y M L 089 C$ & -12.0 & $1.6 \mathrm{E}-03$ & $15.0 \pm 3.1$ & $1.3 \pm 0.3$ & $\mathrm{~ns}$ \\
\hline & LEE1 & -12.1 & $7.0 \mathrm{E}-03$ & $265.8 \pm 82.5$ & $22.0 \pm 5.0$ & Nucleic acid binding \\
\hline & $Y M R 206 \mathrm{~W}$ & -12.2 & $1.8 \mathrm{E}-03$ & $117.1 \pm 25.0$ & $9.6 \pm 1.1$ & ns \\
\hline & $H B N 1$ & -12.3 & $6.5 \mathrm{E}-08$ & $207.3 \pm 1.2$ & $16.8 \pm 3.2$ & Oxidoreductase activity \\
\hline & $S T L 1$ & -27.0 & $1.4 \mathrm{E}-03$ & $2746.6 \pm 579.1$ & $101.9 \pm 34.3$ & Hydrogen symporter activity \\
\hline \multirow[t]{5}{*}{ Xylose } & $Y D R 034 W-B$ & -10.8 & $1.6 \mathrm{E}-03$ & $181.7 \pm 36.9$ & $16.9 \pm 4.6$ & ns \\
\hline & $P A U 15$ & -11.1 & $7.4 \mathrm{E}-04$ & $16.3 \pm 2.7$ & $1.5 \pm 0.6$ & ns \\
\hline & $K D X 1$ & -11.5 & $8.4 \mathrm{E}-06$ & $347.6 \pm 18.8$ & $30.1 \pm 1.6$ & Protein kinase activity \\
\hline & $D A K^{2}$ & -15.1 & $1.1 \mathrm{E}-05$ & $127.0 \pm 7.4$ & $8.4 \pm 1.1$ & Glycerone kinase activity \\
\hline & $A N S 1$ & -28.3 & $1.4 \mathrm{E}-03$ & $14.5 \pm 3.1$ & $0.5 \pm 0.1$ & ns \\
\hline
\end{tabular}

${ }^{\mathrm{a}} p<0.01,>10$-fold, range $>10$.

${ }^{\mathrm{b}}$ Total number of RPKM.

ns, not specific.

Table 4. GSEA using GO biological process on DE genes bygcr2 


\begin{tabular}{|c|c|c|}
\hline & Up-regulated & Down-regulated \\
\hline On glucose & $\begin{array}{l}\text { Translation (6) } \\
\text { Ribosome-related (21) } \\
\text { Nucleotide biosynthesis (1) } \\
\text { Lipid biosynthesis }(5) \\
\text { Antibiotic resistance }(2)\end{array}$ & $\begin{array}{l}\text { Transcriptional regulation }(6) \\
\text { Protein modification and } \\
\text { catabolism (5) } \\
\text { Sugar metabolism (2) } \\
\text { Fatty acid catabolism (4) } \\
\text { Protein transport (4) } \\
\text { Stress response and DNA repair } \\
(7) \\
\text { Iron metabolism (2) } \\
\text { Mitochondrial degradation (1) } \\
\text { Cell division and sporulation (7) } \\
\text { ER-associated protein catabolic } \\
\text { process (1) } \\
\text { Oxidation reduction (1) }\end{array}$ \\
\hline On xylose & $\begin{array}{l}\text { Translation }(2) \\
\text { Sugar metabolism }(\mathbf{3}) \\
\text { Nucleotide and amino acid } \\
\text { biosynthesis }(3) \\
\text { Lipid biosynthesis }(1) \\
\text { Pheromone-related }(2) \\
\text { Metabolic process }(1) \\
\text { ER-associated protein catabolic } \\
\text { process }(1) \\
\text { Oxidation reduction }(\mathbf{1})\end{array}$ & $\begin{array}{l}\text { Protein transport (1) } \\
\text { Cell wall organization }(2)\end{array}$ \\
\hline Both & $\begin{array}{l}\text { Purine nucleotide biosynthetic } \\
\text { process (1) } \\
\text { One-carbon metabolic process (1) }\end{array}$ & Biological_process (1) \\
\hline
\end{tabular}

Numbers in parentheses represent the number of enriched gene sets.

\section{Figure captions}

Fig. 1. Loss-of-function mutation in GCR2 is responsible for the lack of pho13 effect in xylosefermenting $\boldsymbol{S}$. cerevisiae strains. (a) Improved xylose consumption rates by the deletion of PHO13 (pho13 effect) in engineered strains with different strain backgrounds, except for YSX3 and DA24 strains. (b) Genome sequencing results of the YSX3 and L2612 strains and Sanger sequencing results of haploid spores derived from the KSM diploid (YSX3 $\times$ a derivative of D452-2). (c-e) Xylose fermentation profiles by the SR7 strain (control) and its gene deletion mutants (gcr2, pho13, and gcr2 / pho13 ). Fermentation was performed with an initial cell density of $0.5 \mathrm{~g} / \mathrm{L}$ in YP medium containing $40 \mathrm{~g} / \mathrm{L}$ xylose under microaerobic conditions. ${ }^{*} p<0.05$; NS, not statistically significant; nd, not determined.

Fig. 2. Global transcriptional changes induced by gcr2.Hierarchical clustering and multivariate analysis based on Pearson's correlation (a) and principal component analysis (b). DE genes $(p<0.05,>2$-fold) on glucose (c) and on xylose (d) were identified.

Fig. 3. Gene sets and their genes that are oppositely affected by gcr2.

Fig. 4. Transcriptional changes in the central metabolic pathways induced by gcr2 during glucose (a) or xylose (b) metabolism. The fold change in expression in gcr2 mutant relative to that in the wild-type strain is presented. Glyceraldehyde-3P, glyceraldehyde-3-phosphate; Fructose-6P, fructose6-phosphate; Acetyl-CoA, acetyl coenzyme A. 
(a)

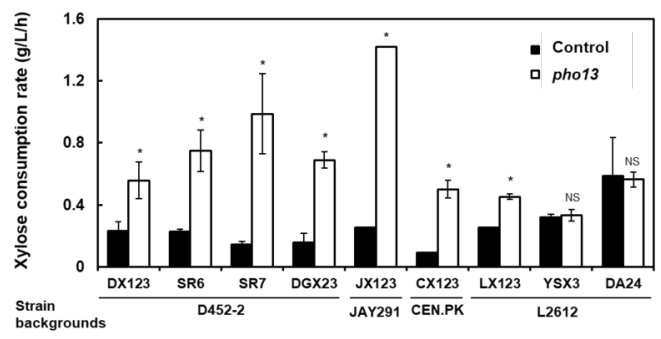

(b)

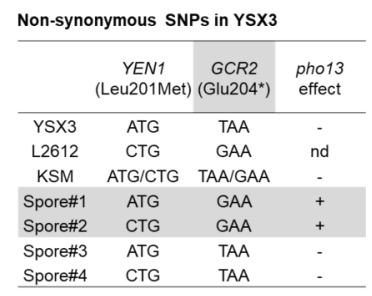

(e)
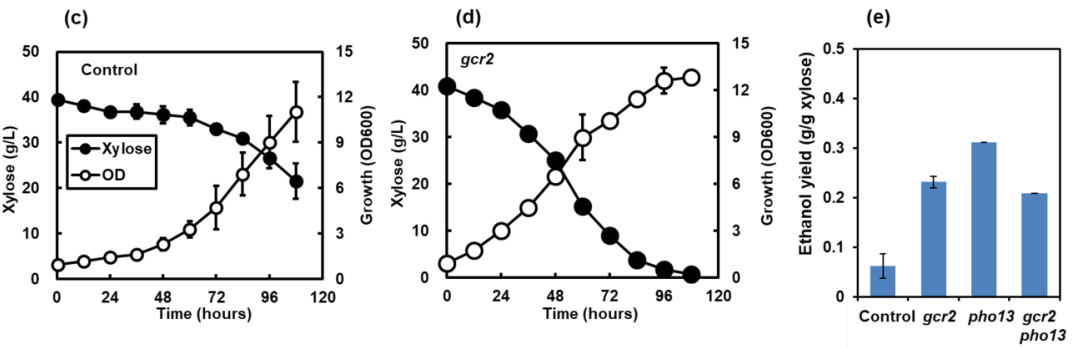

Fig. 1. Loss-of-function mutation in GCR2 is responsible for the lack of pho13 effect in xylose-fermenting $S$. cerevisiae strains.

(a)

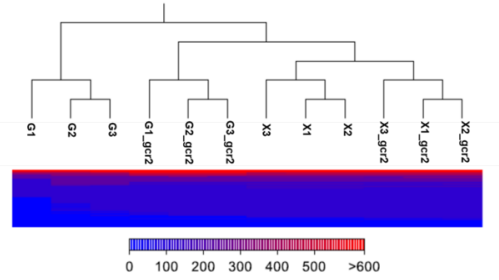

(c)

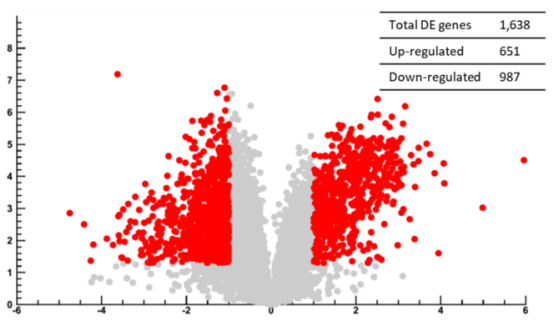

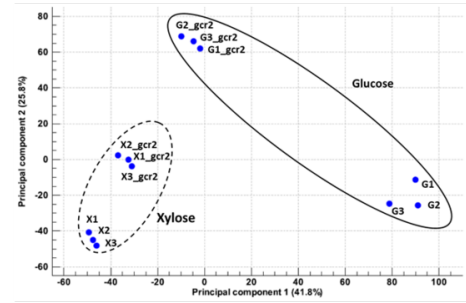

(d)

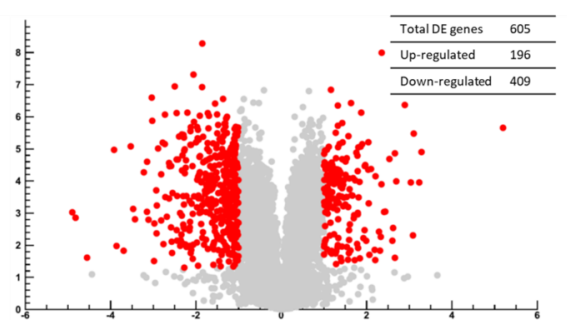

Fig. 2. Global transcriptional changes induced by gcr2. 


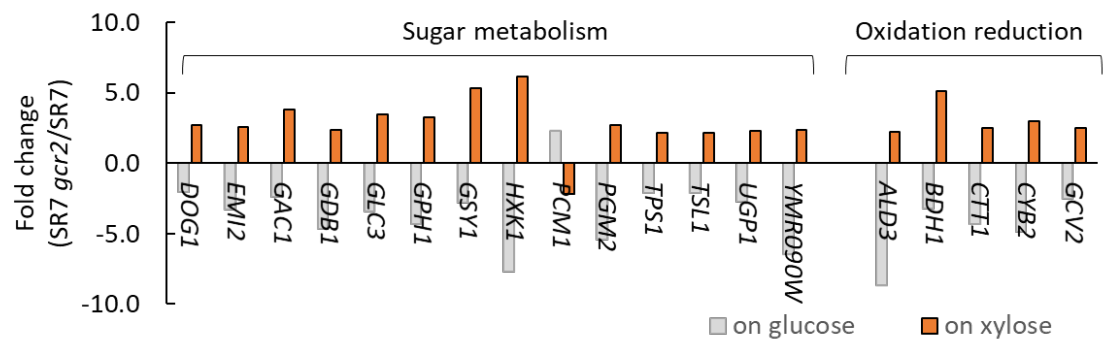

Fig. 3. Gene sets and their genes that are oppositely affected by gcr2.
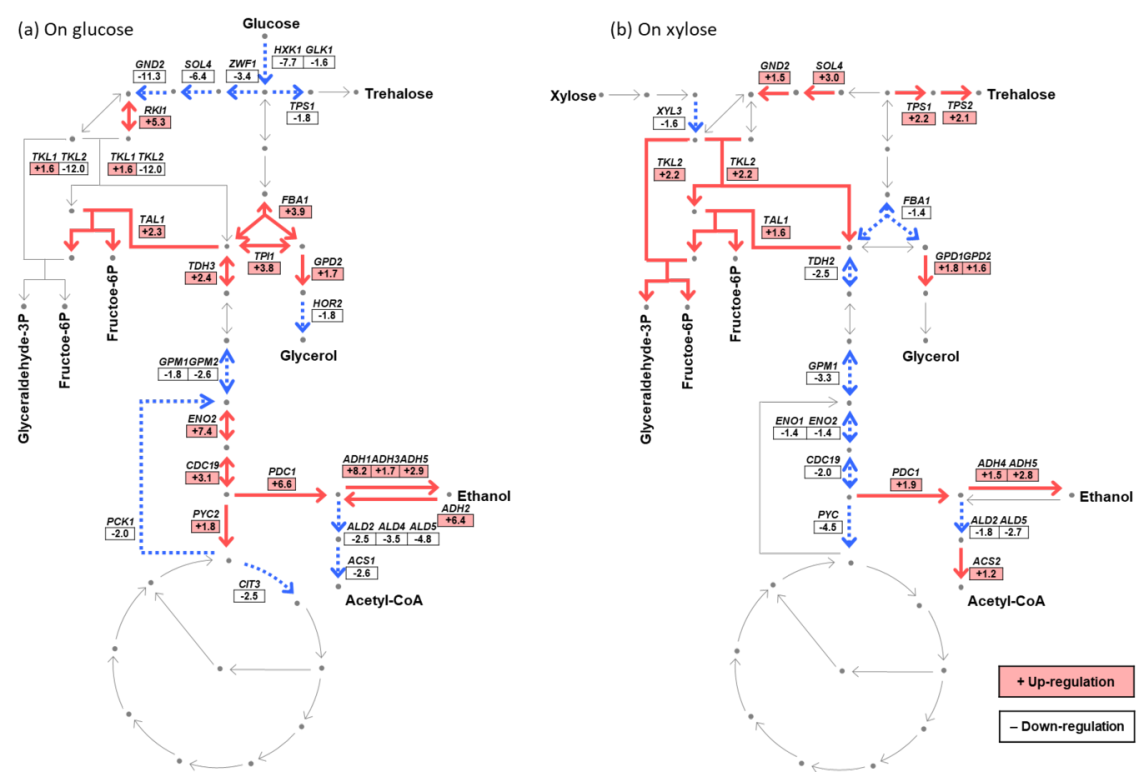

Fig. 4. Transcriptional changes in the central metabolic pathways induced by gcr2 during glucose or xylose metabolism. 